\title{
Desempenho da Força em Idosas Após Duas Intensidades do Exercício Aeróbio
}

\section{Strength Performance in Older Women after Two Intensities of Aerobic Exercise}

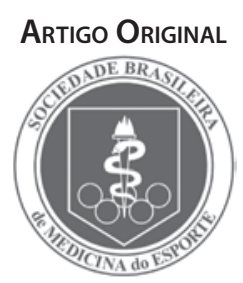

\author{
Adriana Lemos ${ }^{1}$ \\ Roberto Simão 2 \\ Walace Monteiro 3,4 \\ Marcos Polito 5 \\ Jefferson Novaes ${ }^{1,2}$ \\ 1. Universidade Castelo Branco \\ - LABIMH/ PROCIMH - RJ \\ 2. Escola de Educação Física e \\ Desportos - Universidade Federal \\ do Rio de Janeiro (EEFD/UFRJ). \\ 3. Universidade Salgado de Oliveira \\ (UNIVERSO - RJ). \\ 4. Laboratório de Fisiologia do \\ Exercício - Instituto de Ciências \\ da Atividade Física da Aeronáutica \\ (ICAF).
}

5. Universidade Gama Filho (CEPAC).

\section{Endereço para correspondência: Endereço - Escola de Educação Física e Desportos - Universidade Federal do Rio de Janeiro. Departamento de Ginástica. Av. Pau Brasil, 540. Ilha do Fundão. Rio de Janeiro. 21941-590. Email: robertosimao@ig.com.br}

Submetido em 26/07/2006 Versão final recebida em 17/11/2006 Aceito em 28/09/2007

\begin{abstract}
RESUMO
O objetivo do presente estudo foi comparar a influência aguda de duas intensidades de característica aeróbia, sobre o número de repetições numa sessão de treinamento de força (TF) em idosas fisicamente ativas. Oito voluntárias (67,5 $\pm 4,8$ anos; $58,6 \pm 2,2 \mathrm{~kg} ; 156 \pm 5,9 \mathrm{~cm})$ com experiência em ambas atividades, realizaram 20 minutos de caminhada em esteira rolante com intensidades pré-determinadas ( 60 ou $80 \%$ da $F C_{\text {máx }}$ ) e imediatamente após, realizaram uma sessão de TF nos exercícios de pressão de pernas, cadeira extensora e cadeira flexora. Para determinação da carga, foram conduzidos teste e re-teste de 10 repetições máximas (10RM) nos exercícios selecionados, e a $\mathrm{FC}_{\text {máx }}$ foi determinada através de teste de esforço máximo em esteira rolante, com protocolo de Balke-Ware. O número de repetições em cada série após cada intensidade aeróbia foi analisado pela ANOVA de medidas repetidas, seguida do teste post-hoc de Scheffé. O número total de repetições em cada exercício e o número total de repetições em cada seqüência foi analisado pelo teste t-student para amostras dependentes. Os resultados dos testes demonstraram reduções significativas no número total de repetições em toda a sessão de treinamento (somatório de todas as repetições de todos os exercícios). Também foram observadas reduções no número de repetições totais por exercício nas diferentes intensidades do treino de característica aeróbia, a 60 e $80 \%$ da FC máx. Verificou-se também que após o exercício aeróbio realizado a 80\%, todos os avaliados apresentaram maior grau de cansaço, expresso através da percepção subjetiva de esforço. Tal efeito inibitório ficou mais evidenciado através da análise intersequêncial onde foi observado que a $80 \%$ da $\mathrm{FC}_{\text {máx, }}$ ocorreu maior redução do desempenho da força. Conclui-se então, que independentemente das intensidades estudadas, 20 minutos de treinamento aeróbio, podem ser suficientes para provocar redução aguda no desempenho da força em idosas fisicamente ativas.
\end{abstract}

Palavras-chave: treinamento de força, exercícios resistidos, desempenho, treinamento concorrente.

\begin{abstract}
The aim of this study was to compare the acute influence of two intensities of aerobic characteristic on the number of repetitions in a strength training (ST) session in physically active older women. Eight women $(67.5 \pm 4.8$ years; $58.6 \pm 2.2 \mathrm{~kg} ; 156 \pm 5.9 \mathrm{~cm})$ with experience in both activities, volunteered to perform the aerobic activity in both intensities ( 60 or $80 \% H R_{\text {max }}$ ), followed by a training session on leg-press, leg extension and leg curl exercises, at different days. In order to determine the maximal load, 10RM test and re-test were conducted for the selected exercises, and the $\mathrm{HR}_{\max }$. was calculated through the Balke-Ware maximal test protocol. The number of repetitions in each set after aerobic intensity training was analyzed using one-way ANOVA, followed by Scheffé post-hoc test. The total number of repetitions in each exercise and sequence was analyzed by the t-Student test for dependent samples. The results showed significant reductions in the total number of repetitions for all training sessions (sum of all repetitions in all exercises), as well as in the number of repetitions per exercise, after aerobic training intensities of 80 and $60 \%$. We have also verified that after $80 \%$ of $H R_{\max }$. after aerobic exercise, all evaluated subjects showed higher degree of fatigue, expressed by their perceived exertion. Fatigue became more evident when we applied an inter-sequential analysis, which showed a higher reduction in strength training performance when $80 \%$ of $\mathrm{HR}_{\max }$ was applied. We conclude that, regardless the training intensity, 20 minutes of aerobic training may be enough to produce a negative impact on strength training performance of physically active elderly women.
\end{abstract}

Keywords: strength training, resistive exercises, performance, concurrent training.

\section{INTRODUÇÃO}

O envelhecimento é um processo complexo dinâmico e progressivo que envolve a interação de inúmeras variáveis tanto morfológicas como funcionais, que são capazes de influenciar significativamente a forma com a qual alcançamos determinada idade ${ }^{(1,2)}$. Assim, a manutenção da autonomia e independência do idoso vão depender muito do estilo de vida adotado por eles no decorrer deste processo, onde o sedentarismo pode acarretar uma maior incidência de processos patológicos.

O American College of Sports Medicine ${ }^{(2)}$ (ACSM) recomenda a participação de idosos em atividades físicas regulares de exercícios aeróbios e de força, de forma a contribuir para o envelhecimento saudável. Dessa forma, recomenda-se um programa de treinamento de força (TF) que envolva grandes grupamentos musculares, com freqüência de no mínimo duas a três vezes por semana, com sessões que envolvam duas a três séries de cada exercício programado. Já o exercício aeróbio deve ser de intensidade moderada, com uma freqüência semanal de três dias, com duração mínima de 20 minutos e intensidade variando entre 55 a 85\% da freqüência cardíaca máxima $\left(F C_{\text {máx }}\right)$ dependendo do nível de condicionamento do indivíduo ${ }^{(3)}$.

No entanto, o treino simultâneo de exercícios aeróbios e de força em uma mesma sessão, conhecido na literatura como treinamento 
concorrente, têm provocado algumas discussões sobre as adaptações e influências agudas de um treino sobre outro, em função dos diferentes sistemas fisiológicos exigidos em cada treinamento(4).

Em uma revisão, Leveritt et al. ${ }^{(5)}$ relataram que os diferentes mecanismos de adaptação ao treinamento aeróbio contribuíram para a inibição do desenvolvimento da força, e os fenômenos observados foram associados à hipótese crônica ou aguda. A hipótese crônica propõe que o músculo não pode se adaptar metabólica ou morfologicamente para o treinamento concorrente por causa das diferentes exigências orgânicas que são demandadas. A hipótese aguda afirma que o TF é comprometido pela fadiga residual resultante do treinamento de aeróbio prévio ${ }^{(4)}$. Os mecanismos fisiológicos responsáveis por produzir fadiga residual associada com a hipótese aguda parecem estar associados aos parâmetros de treino utilizados no exercício de característica aeróbia ${ }^{(5)}$. Assim, sugere-se que tal efeito inibitório encontrado de forma constante na literatura pode estar associado às atividades aeróbias de longa duração e intensidade ${ }^{(4,5)}$.

Mas apesar de alguns estudos terem examinado os benefícios e comprometimentos de programas que incluem componentes de força e aeróbio em uma mesma sessão de treinamento, apenas dois experimentos ${ }^{(6,7)}$ verificaram tal efeito em idosos fisicamente ativos, e nenhum até o momento verificou os efeitos agudos promovidos pelo treinamento aeróbio em diferentes intensidades sobre uma sessão de TF em idosos. Dessa forma, algumas lacunas podem ser verificadas a respeito do tema proposto em idosos, dentre elas: a diversificação dos tipos de sujeitos investigados, o tipo de intervenção adotado, e a escassez de experimentos que apresentem protocolos de treino similares aos praticados em academias, e em centros de treinamento.

Como ambas atividades são muito procuradas por idosas nas academias como meios de aprimorar a aptidão física, estética e saúde, faz-se necessário o conhecimento da influência aguda de diferentes intensidades de atividade aeróbia sobre o desempenho da força. Com este intuito, buscamos utilizar as intensidades de 60 e $80 \%$ da FC $_{\text {máx. }}$ por serem valores que se inserem dentro da margem preconizada pelo $\mathrm{ACSM}^{(3)}$. Além disso, tais valores podem ser categoricamente definidos como de intensidade alta e baixa, visto que nas academias são comumente utilizados na prescrição de treino para a população em questão. O mesmo critério foi adotado para determinação do tempo de 20 minutos e para a escolha dos exercícios de força.

Cabe ressaltar, que apesar da utilização de duas intensidades de característica aeróbia, a hipótese de influência aguda na capacidade de produção de tensão pode ser relacionada ao fato de ambos exercícios envolverem os mesmos grupamentos musculares. O estudo da influência no desenvolvimento da força nesta população pode ser de grande importância, pois é nesta fase que o desenvolvimento da força implica na autonomia do idoso. Tal conhecimento associa-se também à qualidade e segurança da prescrição do exercício para uma população com características especiais.

Assim, o objetivo do presente estudo foi comparar a influência aguda de duas intensidades de característica aeróbia sobre o número de repetições de uma sessão TF subseqüente em idosas fisicamente ativas.

\section{MATERIAIS E MÉTODOS}

\section{Amostra}

Participaram do estudo oito idosas fisicamente ativas (67,5 \pm 4,8 anos; $58,6 \pm 2,2$ kg; $156 \pm 5,9$ cm) com experiência em TF há pelo menos seis meses com freqüência de três vezes semanais. Todas responderam ao questionário PAR-Q ${ }^{(8)}$ e assinaram um termo de consentimento pósinformado, conforme Resolução 196/96 do Conselho Nacional de Saúde, antes da coleta de dados. O protocolo do estudo foi aprovado pelo comitê de ética institucional da Universidade Castelo Branco (UCB - Rio de Janeiro). Foram adotados os seguintes critérios de exclusão: a) uso de medicamentos que tivessem influência sobre o comportamento das respostas cardiovasculares; b) existência de problemas osteomioarticulares que pudessem influenciar na realização dos exercícios propostos.

\section{PROTOCOLO}

\section{Coleta dos dados}

A coleta de dados foi efetuada em cinco dias alternados com 48 a 72 horas de intervalo. Na primeira visita ao laboratório, foram realizadas as medidas antropométricas e o teste de esforço cardiopulmonar. No segundo e terceiro dias, foram conduzidos testes de 10 repetições máximas (10RM) nos exercícios selecionados (teste e re-teste). Nos dois últimos dias de coleta, realizaram-se sessões de TF após o trabalho aeróbio nas intensidades determinadas (60 ou 80\% da FC máx.).

\section{Teste de esforço}

O teste foi realizado em esteira rolante, através de protocolo de Balke-Ware, devido aos seus baixos estágios de progressão que são mais adequados para os indivíduos idosos ${ }^{(3)}$. A inclinação da esteira foi aumentada em $1 \%$ a cada minuto até a exaustão do avaliado e não foi permitido segurar nas barras lateral ou frontal da esteira, mantendo-se a velocidade constante em 3,4 kph. Como critério de teste máximo foram observados os seguintes aspectos: ausência ou pequena variação da freqüência cardíaca em esforço apesar do aumento da carga do exercício, exaustão voluntária máxima, bem como qualquer sinal ou sintoma limitante. Todos os indivíduos eram adaptados ao trabalho em esteira rolante, e durante a realização do teste foram incentivados a atingir a exaustão máxima. A temperatura da sala para condução dos testes foi mantida entre 21 e $23^{\circ} \mathrm{C}$. Todos os testes foram realizados por médicos cardiologistas. Monitoraram-se as seguintes variáveis antes e durante cada estágio alem do pós-teste: freqüência cardíaca, pressão arterial, percepção subjetiva de esforço (PSE), eletrocardiograma e sintomas clínicos. O horário do teste foi similar ao horário do treinamento, que era compreendido entre oito e nove horas da manhã.

\section{Teste de 10RM}

O procedimento adotado na aplicação do teste $10 \mathrm{RM}^{(9)}$ respeitou na seguinte ordem: pressão de pernas $45^{\circ}$ (PP), cadeira extensora (CE) e cadeira flexora (CF). Os exercícios foram selecionados devido à sua disseminação em centros de treinamento e facilidade de execução, e os aparelhos utilizados foram da marca Selection TechnoGym ${ }^{\circledR}$.

Visando reduzir a margem de erro nos testes de 10RM, foram adotadas as seguintes estratégias ${ }^{(10)}$ : a) instruções padronizadas foram fornecidas antes do teste, de modo que o avaliado estivesse ciente de toda a rotina que envolvia a coleta de dados; b) o avaliado foi instruído sobre a técnica de execução do exercício; c) o avaliador estava atento quanto à posição adotada pelo praticante no momento da medida, pois pequenas variações no posicionamento das articulações envolvidas no movimento poderiam acionar outros músculos, levando a interpretações errôneas dos escores obtidos; d) estímulos verbais foram realizados a fim de manter alto o nível de estimulação. Os intervalos entre as tentativas em cada exercício durante o teste de 10RM foram fixados entre dois a cinco minutos ${ }^{(9)}$. Após obtenção da carga em um determinado exercício, intervalos não inferiores a 10 minutos foram dados, antes de passar-se ao teste no exercício seguinte.

Após a obtenção das cargas máximas no teste de 10RM, os indivíduos descansaram por 48 horas e foram reavaliados para obtenção da reprodutibilidade da carga no teste (teste e re-teste). Considerouse como 10RM a maior carga estabelecida em ambos os dias com diferença menor que 5\%. Caso a diferença das cargas obtidas fossem maior que 5\% um novo teste e re-teste eram realizados. Nos intervalos entre as sessões de testes não foi permitida a realização de exercícios para não interferir nos resultados obtidos. O horário do teste e re-teste foi similar ao horário de treinamento. 


\section{Procedimento de coleta}

Após a obtenção das cargas em 10RM, duas sessões de treinamento com intervalo de 48 horas foram executadas em duas formas seqüenciais. A seqüência A (SEQA) consistia na realização de uma sessão de treino aeróbio na esteira rolante com duração de 20 minutos a 60\% da $F C_{\text {máx, }}$ seguido de um TF nos três exercícios selecionados em três séries com cargas para 10RM. Na seqüência B (SEQB) o procedimento foi similar a SEQA diferenciando-se o trabalho aeróbio prévio na esteira rolante a $80 \%$ da $\mathrm{FC}_{\text {máx. }}$ A ordenação dos exercícios de força foi o mesmo nas duas seqüências: PP, CE e CF. A inclusão dos indivíduos na realização das seqüências foi definida pelo delineamento alternado.

Ao final da realização do trabalho aeróbio, o avaliado era questionado quanto à sua PSE adotando-se como referência a escala de Borg CR10 ${ }^{(11)}$, que era apresentada ao avaliado sempre ao final do treinamento aeróbio, para que o mesmo definisse um valor na escala. Um intervalo de dois minutos foi permitido antes de iniciar o TF.

Após o treino aeróbio a 60 ou $80 \%$ da $\mathrm{FC}_{\text {máx, }}$ os voluntários realizaram três séries de cada exercício com cargas de 10RM até a falha concêntrica, com intervalo de 90 segundos entre as séries e dois minutos entre os exercícios. Para execução do máximo número de repetições até exaustão voluntária, o avaliador motivou os voluntários. Em cada série, mensurou-se o número máximo de repetições realizadas. Todos os testes e sessões de treinos foram realizados entre oito e nove horas da manhã com o objetivo de respeitar a rotina de treino das voluntárias.

\section{Tratamento Estatístico}

O número de repetições em cada série após cada intensidade aeróbia foi analisado pela ANOVA de medidas repetidas, seguida do teste post-hoc de Scheffé. O número total de repetições em cada exercício e o número total de repetições em cada seqüência foi analisado pelo teste t-student para amostras dependentes. A PSE foi analisada pelo teste não-paramétrico de Wilcoxon.

A determinação da reprodutibilidade nas cargas para 10RM foi realizada através do coeficiente de correlação intra-classe, adicionada ao teste $t$ de student pareado. Em todos os casos adotou-se nível de significância estatística $p<0,05$. Os dados foram tratados no software Statistica 5.5 (Statsoft, USA).

\section{RESULTADOS}

O volume total de repetições (somatório de todas as repetições de todos os exercícios) foi estatisticamente mais elevado após o exercício aeróbio com $60 \%$ da $\mathrm{FC}_{\text {máx }}$. $(78,3 \pm 1,7$ repetições) em relação ao exercício realizado com $80 \%$ da $\mathrm{FC}_{\text {máx. }}$ (61,1 $\pm 6,6$ repetições) (Figuras 1, 2 e 3). As Figuras 1, 2 e 3 também apresentam a comparação do número de repetições em cada série (inter-sequência) dos exercícios PP, CE e CF, respectivamente, após a atividade aeróbia com intensidade de 60\% da $F C_{\text {máx }}$ e $80 \%$ da $F C_{\text {máx. }}$

A comparação intra-seqüência mostrou um comportamento semelhante para todos os exercícios, independentemente da atividade aeróbia prévia. Em todos os casos, houve uma redução do número de repetições no decorrer das séries. Dessa forma, foi observada diferença significativa entre a primeira e segunda série, entre a primeira e a terceira e entre a segunda e a terceira séries. A única exceção foi o exercício PP realizado após a atividade aeróbia a $60 \%$ da $\mathrm{FC}_{\text {máx, onde }}$ as diferenças ocorreram somente entre a primeira e a segunda, e entre a primeira e a terceira séries (Tabela 1).

Os resultados (coeficientes de correlação intra-classe) referentes à reprodutibilidade das cargas obtidas nos testes de 10RM foram: PP $r=0,96 ; C E r=0,94$ e CF r=0,96. Em adição, o teste-t student pareado não demonstrou diferença significativa entre as cargas obtidas para 10RM em cada exercício $(p<0,05)$. A PSE foi significativamente mais elevada após a atividade aeróbia realizada com $80 \%$ da $\mathrm{FC}_{\text {máx }}$ (mediana 6) em relação à atividade com $60 \%$ da $\mathrm{FC}_{\text {máx }}$ (mediana 5).

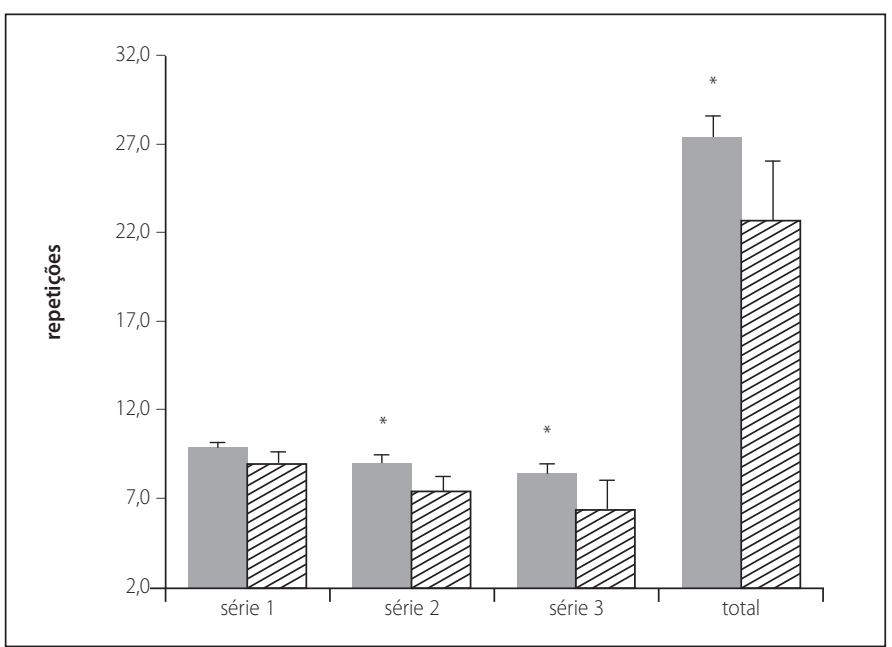

Figura 1. Comportamento do número de repetições em cada série do exercício PP realizado após a atividade aeróbia com intensidade de $60 \%$ da $\mathrm{FC}_{\text {máx }}$ (coluna cinza) e $80 \%$ da $\mathrm{FC}_{\text {máx. }}$ (coluna listrada).

* diferença significativa em relação ao exercício realizado após a intensidade de $80 \%$ da $\mathrm{FC}_{\text {máx. }}$

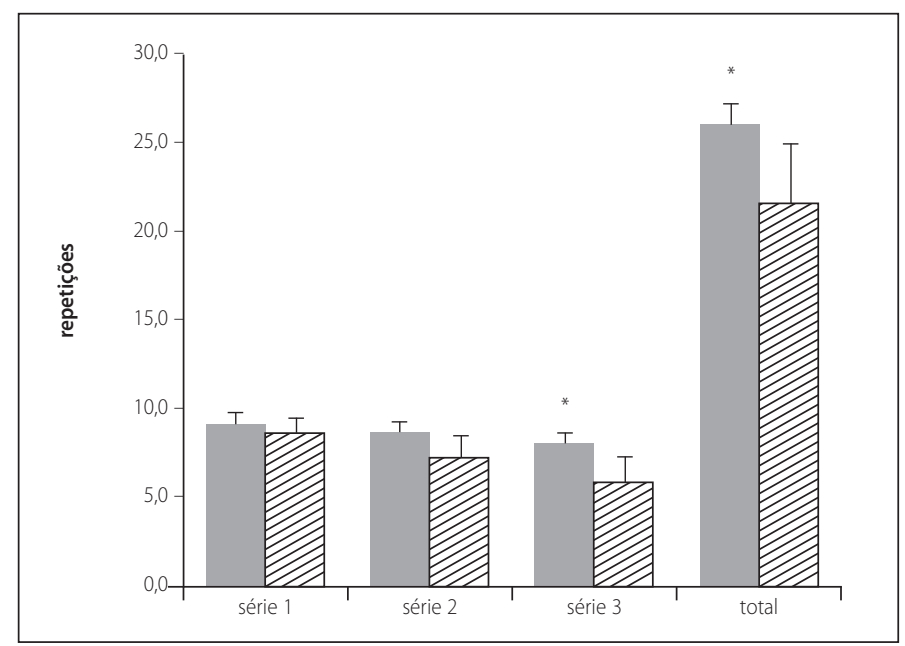

Figura 2. Comportamento do número de repetições em cada série do exercício CE realizado após a atividade aeróbia com intensidade de $60 \%$ da $\mathrm{FC}_{\text {máx }}$ (coluna cinza) e $80 \%$ da FC $_{\text {máx }}$ (coluna listrada).

* diferença significativa em relação ao exercício realizado após a intensidade de 80\% da $\mathrm{FC}_{\text {máx} \text {. }}$

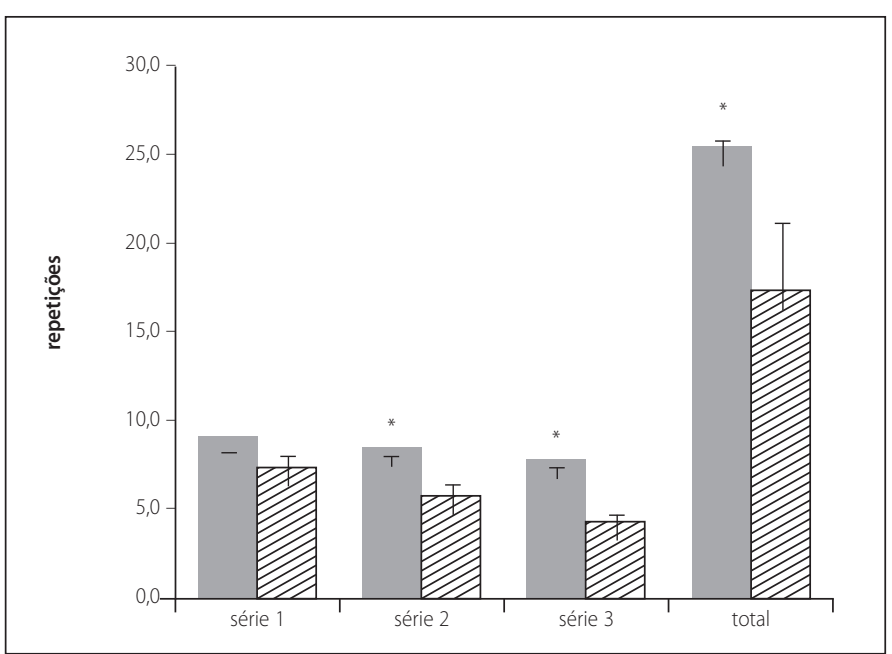

Figura 3. Comportamento do número de repetições em cada série do exercício CF realizado após a atividade aeróbia com intensidade de $60 \%$ da FC $_{\text {máx. }}$ (coluna cinza) e $80 \%$ da FC $_{\text {máx. }}$ (coluna listrada).

* diferença significativa em relação ao exercício realizado após a intensidade de $80 \%$ da $\mathrm{FC}_{\text {máx. }}$ 
Tabela 1. Comportamento do número de repetições intra-seqüência em cada série dos exercícios após a atividade aeróbia com intensidade de 60 e 80\% da FC máx.

\begin{tabular}{|c|c|c|c|c|c|c|}
\hline & \multicolumn{3}{|c|}{$60 \%$} & \multicolumn{3}{|c|}{$80 \%$} \\
\hline & PP & CE & CF & PP & CE & $C F$ \\
\hline $1^{\text {a }}$ série & $9,9 \pm 0,4^{* @}$ & $9,3 \pm 0,5^{* @}$ & $9,0 \pm 0,0^{* @}$ & $8,9 \pm 0,8^{* @}$ & $8,5 \pm 0,9 * @$ & $7,3 \pm 1,7^{* @}$ \\
\hline $2^{\mathrm{a}}$ série & $9,0 \pm 0,5$ & $8,6 \pm 0,5^{\#}$ & $8,4 \pm 0,5^{\#}$ & $7,4 \pm 0,9^{\#}$ & $7,1 \pm 1,2^{\#}$ & $5,6 \pm 1,4^{\#}$ \\
\hline $3^{a}$ série & $8,5 \pm 0,5$ & $8,0 \pm 0,5$ & $7,6 \pm 0,7$ & $6,4 \pm 1,7$ & $5,9 \pm 1,4$ & $4,1 \pm 1,4$ \\
\hline Total & $27,4 \pm 1,2$ & $25,9 \pm 1,2$ & $25,0 \pm 1,2$ & $22,6 \pm 3,3$ & $21,5 \pm 3,3$ & $17,0 \pm 4,2$ \\
\hline
\end{tabular}

PP - Pressão de pernas

CE - Cadeira extensora.

CF - Cadeira flexora.

* Diferença significativa entre a primeira e segunda série.

@ Diferença significativa entre a primeira e terceira série.

\# Diferença significativa entre a segunda e terceira série.

\section{DISCUSSÃO}

Os principais resultados aqui apresentados demonstraram reduções significativas no número total de repetições em toda a sessão de treinamento (somatório de todas as repetições de todos os exercícios), e no número de repetições totais por exercício, quando executados após exercício aeróbio em 80\% quando comparado a 60\%. Ao analisar de forma inter-sequêncial verificou-se nas respectivas séries, uma tendência a redução mais acentuada do número de repetições após o treinamento conduzido a 80\% da FC máx. A comparação intra-seqüência mostrou um comportamento semelhante para todos os exercícios, onde foi observada uma redução significativa do número de repetições no decorrer das séries independentemente da intensidade de característica aeróbia adotada. Já no exercício PP realizado após a atividade aeróbia de 60\% da $\mathrm{FC}_{\text {máx }}$ ocorreram diferenças significativas apenas entre a primeira e a segunda, e entre a primeira e a terceira séries.

O efeito deletério observado no desempenho do TF através do número de repetições, pode estar relacionado à fadiga residual ocasionada pela solicitação dos mesmos grupamentos musculares em ambas as formas de treinamento. Dentre as causas periféricas da fadiga residual aguda pode-se citar o acúmulo de metabólitos (fosfato inorgânico, ácido lático, amônia) e esgotamento de substratos de energia como ATP, fosfato de creatina e glicogênio muscular. Níveis elevados de lactato no sangue são evidentes após o exercício de resistência de alta intensidade ${ }^{(5)}$. Tais fatores podem ser responsáveis por promover a alteração no processo excitação - contrátil , ou seja, podem interferir na capacidade de produção de tensão do músculo envolvido no exercício aeróbio prévio.

Os mecanismos de fadiga certamente influenciaram significativamente nos resultados, principalmente quando comparamos o volume total de treinamento. No entanto, quando comparamos as médias de repetições entre as séries, verificamos que na primeira série da PP, na primeira e segunda série da CE, e na primeira série da CF não houve diferença significativa entre as intensidades estudadas, mas sim uma tendência ao decréscimo no número de repetições após a atividade

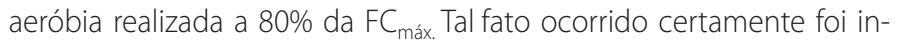
fluenciado pela maior intensidade de esforço promovido pela atividade aeróbia onde também foram relatados maiores valores da PSE. Mas vale ressaltar, que a interferência no número de repetições em cada série por exercício, não foi decorrente exclusivamente dos mecanismos de fadiga promovidos pela atividade aeróbia prévia, mas também pelo efeito da fadiga resultante da realização de cada série. Assim, ao analisarmos toda a seqüência da sessão de TF, observamos uma tendência a um menor volume de treinamento após a intensidade de 80\% da FC $C_{\text {máx }}$ provavelmente em função do somatório da atividade aeróbia prévia associada a fadiga promovida após cada série.
Já a diferença significativa verificada no volume total na comparação das duas intensidades, 60 e 80 \% da $\mathrm{FC}_{\text {máx. }}$ pode estar associada à não utilização da intensidade mais alta no treino aeróbio diário das voluntárias, pois a maioria das idosas avaliadas realizavam o treinamento aeróbio mais próximo do limiar inferior.

Tais resultados são um importante fator a ser observado na otimização das respostas do TF, pois a redução no volume de treinamento, pode comprometer ganhos de força e hipertrofia, como já foi evidenciado em estudos sobre o treinamento concorrente ${ }^{(12-16)}$. Sporer e Wenger ${ }^{(17)}$ observaram que após diferentes períodos de recuperação em dois tipos de treinamento aeróbio distintos, sub-máximo e intervalado, realizados em ciclo ergômetro, houve uma redução no volume total de repetições a 75\% de 1RM no leg press, embora o mesmo não tenha ocorrido com o exercício supino. Tal estudo investigou se o tipo e a intensidade do treinamento aeróbio empregado influenciavam nos exercícios de força em uma mesma sessão.

Leveritt e Abernethy ${ }^{(18)}$ também verificaram o efeito agudo de um treinamento aeróbio de alta intensidade sobre o subseqüente TF envolvendo exercícios de agachamento e extensão de pernas. 0 treinamento aeróbio era realizado em ciclo ergômetro entre 40 a 100\% do $\mathrm{VO}_{2}$ de pico. $\mathrm{O}$ exercício agachamento envolvia três séries a $80 \%$ de $1 \mathrm{RM}$, enquanto a cadeira extensora no aparelho isocinético consistia em 5RM em cinco diferentes velocidades de execução. Foram verificadas significantes reduções em ambos os exercícios.

As evidências encontradas convergem com o estudo de Abernethy ${ }^{(19)}$, que verificou a influência de dois tipos de treinamento aeróbio, contínuo de baixa intensidade e intervalado de alta intensidade, em teste subseqüente de força realizado no exercício de extensão de pernas em aparelho isocinético. A força era avaliada em 10 velocidades de contração máxima. Ambas as formas de treinamento aeróbio prévio influenciaram na redução das contrações máximas de forma significativa. Em contraposição aos estudos citados Leverrit et al.(20) demonstraram que o desempenho na extensão de perna na sessão de força, conduzido em distintos tipos de trabalho muscular, não foi afetada de oito a 32 horas, após 50 minutos no ciclo ergômetro (70$110 \%$ da potência crítica).

As divergências nos resultados dos presentes estudos podem estar associadas às metodologias empregadas em cada experimento, como por exemplo, as características da amostra avaliada, as formas de trabalho muscular solicitadas, a intensidade do esforço requeridos no exercício aeróbio e de força, o tamanho da musculatura, os diferentes instrumentos de medidas e o tempo pós-esforço em que foi avaliada a força. No presente experimento foram utilizados exercícios em resistência dinâmica invariável, sendo todos para membros inferiores em uma sessão de TF. A intensidade do esforço na força foi determinada através do teste de 10RM realizado em três diferentes exercícios, onde o grau de fadiga foi evidenciado pela redução do número de repetições nas três séries de cada exercício. Tal metodologia foi utilizada com intuito de aproximar o experimento da realidade das idosas que praticam regularmente tais atividades em academias.

Apesar das diferenças metodológicas verificadas nos estudos abordados e no atual experimento, os dados do presente trabalho corroboram com outros estudos que verificaram influência negativa do treinamento aeróbio prévio no desenvolvimento da força ${ }^{(17-20)}$. Ainda assim, vale ressaltar algumas semelhanças referentes ao treino aeróbio nos referidos estudos. Abernethy ${ }^{(19)}$ aplicou um treino aeróbio contínuo de baixa intensidade e outro intervalado de alta intensidade, enquanto Sporer e Wenger ${ }^{(17)}$, testaram a influência de intervalados de alta intensidade e contínuo sub-máximo conduzido em cicloergômetro no desempenho da força. Portanto, pode-se verificar que existe um 
foco comum nos trabalhos supracitados em relação ao atual, no qual o objetivo foi testar duas situações de treino aeróbio, classificadas como baixa ( $60 \%$ da $\mathrm{FC}_{\text {máx. }}$ ) e alta intensidade ( $80 \%$ da $F C_{\text {máx. }}$ ).

Outro fato observado em nossos resultados foi a redução do número de repetições de forma significativa, de acordo com a evolução das séries nos respectivos exercícios. Tal fenômeno nos leva a perceber que a adoção do intervalo de 90 segundos entre as séries e dois minutos entre os exercícios não foi suficiente para manter o número de repetições máximas com as cargas obtidas no teste de 10RM. Tal comportamento foi observado em todos os exercícios investigados, sendo mais evidenciado após o treinamento aeróbio a $80 \%$ da $\mathrm{FC}_{\text {máx }}$. onde todos os avaliados apresentaram maior grau de cansaço, expresso através da PSE.

O comportamento apresentado nos resultados do presente experimento, corroboram a hipótese do efeito da fadiga residual localizada no músculo treinado concorrentemente ${ }^{(18,20)}$, que costuma se apresentar através da diminuição da força ou potência muscular em resposta a um esforço voluntário gerando assim uma redução do desempenho. Isso nos faz pensar que a fadiga proporcionada pelo treinamento aeróbio, mesmo em indivíduos idosos que possuem baixo $\mathrm{VO}_{2}$ de pico, pode afetar o desempenho na força.

Dentre as diferenças encontradas nos estudos que abordaram a influência aguda de uma sessão de exercício aeróbio prévio ao de força, a principal se refere à população voluntária. No presente trabaIho optou-se por idosas fisicamente ativas, com idade entre 60 e 75 anos, enquanto nos demais foram indivíduos jovens. Portanto, cabe

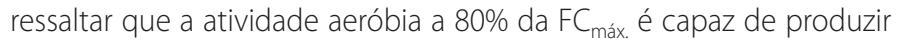
maior efeito negativo no desempenho da força, quando comparado a uma intensidade menor de $60 \%$ da $\mathrm{FC}_{\text {máx, }}$ principalmente na amostra estudada.

Outra diferença refere-se ao padrão de exercício aeróbio adotado, onde nos estudos citados optou-se pelo ciclo-ergômetro ${ }^{(17-19)}$, enquanto na presente investigação, utilizou-se a esteira ergométrica. No que se refere ao treino subseqüente de força, as diferenças encontradas residem sobre os tipos de equipamentos utilizados, testes de carga, séries e exercícios avaliados.

Embora até o momento não haja nenhum trabalho sobre o efeito agudo do treino aeróbio no TF envolvendo idosas ativas fisicamente, vale ressaltar que apenas dois trabalhos ${ }^{(6,7)}$ verificaram o efeito crônico do treinamento concorrente de aeróbio e força em relação aos seus benefícios para a otimização do treinamento funcional na terceira idade.

Algumas limitações do presente estudo podem ser destacadas, tais como: o número reduzido de voluntários composto por oito indivíduos devido à dificuldade de encontrar idosas que não fizessem uso de medicamentos com ação cardiovascular, e ainda a ausência da ergoespirometria na realização dos testes de esforço máximo, importante para fornecer variáveis ventilatórias úteis na quantificação da intensidade do esforço. Mas cabe também ressaltar que o presente estudo teve seu foco voltado para a realidade de treinamento das idosas fisicamente ativas em academias, o que talvez não tenha sido o objetivo central dos outros autores.

Em conclusão, com base nos dados obtidos pôde-se observar que tanto a $60 \%$ quanto a $80 \%$ da $\mathrm{FC}_{\text {máx. }}$ houve influência negativa no desempenho do TF em relação ao volume total de treinamento. Já no que diz respeito a análise intersequências foi observado que a $80 \%$ da FC máx. ocorreu maior redução do desempenho da força. Quanto ao volume adotado, pôde-se perceber que 20 minutos na esteira ergométrica são suficientes para provocar redução no desempenho da força em idosas.

Estudos futuros poderiam envolver maior número de sujeitos em uma sessão de treino de força que envolvesse outros grupamentos musculares. O treino aeróbio poderia ser verificado em períodos maiores de tempo ou em outras intensidades de esforço. A utilização da ergoespirometria no treino aeróbio prévio seria um importante indicador para determinar a intensidade do treino para cada indivíduo, além de serem valiosos parâmetros de discussão. A utilização da eletromiografia seria interessante para mapear nível de comprometimento muscular dos grupamentos envolvidos em ambas as formas de treinamento. A verificação dos efeitos crônicos desta forma de treinamento em idosos seria também de grande importância para nortear a prescrição de exercícios para a população estudada.

Todos os autores declararam não haver qualquer potencial conflito de interesses referente a este artigo.

\section{REFERÊNCIAS BIBLIOGRÁFICAS}

1. Nóbrega ACL, Freitas EV, Oliveira MAB, Leitão MB, Lazzoli JK, Nahas RM, et al. Posicionamento Oficial da Sociedade Brasileira de Medicina do Esporte e da Sociedade Brasileira de Geriatria e Gerontologia: atividade física e saúde no idoso. Rev Bras Med Esporte 1999; 5: 207-11.

2. American College of Sports Medicine. Position stand on the recommended quantity and quality of exercise for developing and maintaining cardiorespiratory and muscular fitness, and flexibility in adults. Med Sci Sports Exerc 1998; 30: 975-91.

3. American College of Sports Medicine. Diretrizes do ACSM para os testes de esforço e sua prescrição. Rio de Janeiro: Guanabara Koogan, 2003.

4. Docherty D, Sporer B. A proposed model for examining the interference phenomenon between concurrent aerobic and strength training. Sports Med 2000; 30: 385-94.

5. Leveritt M, Abernethy P. Acute effects of high-intensity endurance exercise on subsequent resistance activity. J Strength Cond Res 1999; 3: 47-51.

6. Wood RH, Reyes R, Welsch MA, Favaloro-Sebastier M, Mathew LC, Johnson LG, et al. Concurrent cardiovascular and resistance training in healthy older adults. Med Sci Sports Exerc 2001; 33: 1751-8.

7. Takeshima N, Rogers ME, Islam MM, Yamauchi T, Watanabe E, Okada Al. Effect of concurrent aerobic and resistance circuit exercise training on fitness in older adults. Eur.Journal Apply Physiol 2004; 93: 173-82

8. Shephard RJ. PAR-Q: Canadian home fitness test and exercise screening alternatives. Sports Med 1988; 5: 185-95.

9. Bachle TR, Earle RW. Essentials of strength training and conditioning. Champaign: Human Kinetics, 2000.

10. Monteiro W, Simão R, Farinatti PTV. Manipulação na ordem dos exercícios e sua influência sobre o número de repetições e percepção subjetiva de esforço em mulheres treinadas. Rev Bras Med Esporte 2005; 11: 146-50
11. Borg G. Perceived exertion and pain scales. Champaign: Human Kinetics, 1998.

12. Dudley GA, Djamil R. Incompatibility of endurance and strength training modes of exercises. J Appl Physiol 1985; 59: 1446-51.

13. Hunter $G$, Demment $R$, Miller D. Development of strength and maximum oxygen uptake during simultaneous training for strength and endurance. J Sports 1987; 27: 269-75.

14. Hennessy $L C$, Watson AWS. The interference effects of training for strength and endurance simultaneously. J Strength Cond Res 1994; 8: 12-9.

15. Kraemer WJ, Patton JF, Gordon SE, Harman EA, Deschenes MR, Reynolds K, et al. Compatibility of high-intensity strength and endurance training on hormonal and skeletal muscle adaptations. J Appl Physiol 1995; 78: 976-89.

16. Hakkinen $\mathrm{K}$, Alen M, Kraemer WJ, Gorostiaga E, Izquierdo M, Rusko H, et al. Neuromuscular adaptations during concurrent strength and endurance training versus strength training. Eur.J Apply Physiol 2003; 89: 42-52.

17. Sporer $B C$, Wenger $H A$. Effects of aerobic exercise on strength performance following various periods of recovery. J Strength Cond Res 2003; 17: 638-44.

18. Leveritt M, Abernethy PJ, Barry B, Logan PA. Concurrent strength and endurance: a rewiew. Sports Med 1999; 28: 413-27.

19. Abernethy PJ. Influence of acute endurance activity on isokinetic strength. J Strength Cond Res 1993; 7: 141-6.

20. Leveritt M, Mac Laughlin, Abernethy PJ. Changes in leg strength 8 and 32 hours after endurance exercise. J sport Sci 2000; 18: 865-79. 